Voix et Images

voixetimages

\title{
Lascaux, les limbes et autres lieux
}

\section{André Brochu}

Volume 12, numéro 1 (34), automne 1986

Québec-Amérique latine

URI : https://id.erudit.org/iderudit/200615ar

DOI : https://doi.org/10.7202/200615ar

Aller au sommaire du numéro

Éditeur(s)

Université du Québec à Montréal

ISSN

0318-9201 (imprimé)

1705-933X (numérique)

Découvrir la revue

Citer cet article

Brochu, A. (1986). Lascaux, les limbes et autres lieux. Voix et Images, 12(1),

131-140. https://doi.org/10.7202/200615ar d'utilisation que vous pouvez consulter en ligne.

https://apropos.erudit.org/fr/usagers/politique-dutilisation/ 


\title{
Poésie
}

\section{Lascaux, les limbes et autres lieux}

\author{
par André Brochu, Université de Montréal
}

La littérature est soumise aux sautes de vent de la mode, parfois déplorables mais normales. C'est ainsi que les problématiques se renouvellent, que les styles évoluent. Après avoir été pendant des années la puissance montante, la génération des Herbes rouges (ou de la Barre du jour) est aujourd'hui contestée et remet elle-même en cause certaines valeurs un peu bizarres, tel l'illisible. Ce qui étonne cependant, c'est que l'opposition ne vienne pas d'un autre groupe d'âge. Le mécanisme de la succession des générations semble enrayé.

En conséquence, les arguments qu'on voit opposer à la Barre du jour sont en quelque sorte antérieurs aux positions remises en cause et ne font aucunement avancer la théorie ni la pratique littéraires. On se croirait en politique, où Bourassa succède à Lévesque qui succédait à Bourassa.

Le malaise actuel tient aussi au décalage, que j’ai maintes fois signalé, entre l'offre et la demande. Un nombre croissant de bons livres est offert à un public lecteur qui rétrécit comme peau de chagrin. Les gouvernements maintiennent, à coups de subventions, une activité littéraire qui s'élabore de plus en plus en vase clos, loin du client.

La solution n'est certes pas de demander aux écrivains une limitation volontaire de leur production, comme si chacun avait son petit effort à faire pour l'assainissement du marché du livre, mais d'amener les éditeurs à tabler davantage sur la qualité. Il n'y a rien de gênant en soi dans l'abondance d'un Charron, d'un Beausoleil, dans les nombreuses publications de la Nouvelle Barre du jour. On ferait mieux de s'en prendre à la prolifération des publications médiocres, qui fleurissent sous le couvert de toutes esthétiques.

De tous les écrivains de la génération des Herbes rouges, qui en compte de remarquables, Normand de Bellefeuille est certainement l'un des plus importants, par la qualité de sa réflexion théorique et critique comme par l'originalité de son inspiration proprement créatrice. Lascaux ${ }^{1}$, qu'il fait paraître deux ans après son très beau Livre du devoir ${ }^{2}$ (prix Émile Nelligan 1984), ne le cède en rien à ses ouvrages antérieurs pour la rigueur et la générosité de la pensée.

Je parle de pensée même s'il s'agit de "fiction" car une souveraine intelligence orchestre les expressions de la sensibilité et les fastes de l'imagination en une composition qui s'adresse à toutes les facultés de l'esprit. On est, devant les proses et les poésies de Lascaux, comme devant les saisissantes images de la grotte où défilent les formes de la vie animale, les scènes de 
chasse, la représentation émouvante de l'ancêtre, bref tout un vécu archaïque qui permet d'interroger d'un point de vue radical l'aventure humaine.

Cette dimension philosophique n'est pas absente, loin de là, de la méditation de de Bellefeuille, mais elle reste implicite; au premier plan, on trouve plutôt un extraordinaire quatuor de femmes, la mère et les tantes de cet enfant de neuf ans auquel s'identifie le narrateur. Fascinées par Lascaux qu'elles se sont rendu visiter, sans succès puisque la grotte est depuis longtemps fermée au public, elles se réunissent périodiquement pour regarder les trente diapositives qu'elles ont achetées sur les lieux et pour se souvenir des péripéties du voyage. Un climat de douce hystérie, depuis le poker initial jusqu'à la photo de groupe finale, règle les rapports entre ces dames qu'on dirait issues d'un roman de Proust ou d'Éthier-Blais. Un mélange subtil de tendresse et d'ironie nous les fait voir dans leur insondable vérité, individualités finement dessinées sur fond de temps, d'angoisse, de mort. L'enfant, au milieu d'elles et des projections de bêtes fabuleuses, se tait, comme à l'écoute du destin.

Il serait trop long de décrire le dispositif formel, qui utilise quatre discours (ou écritures) différents, les ordonne avec précision, sans excès de rationalité. Plus que jamais, de Bellefeuille rend inopérante la distinction entre prose et poésie, sans pour autant mélanger l'une et l'autre mais en les mettant en tension dialectique dans une écriture qui en redistribue les traits.

Résultat: un livre émouvant, intelligent, drôle, tragique, sans graisse lyrique ni mélasse humaniste, une magnifique provocation à rêver l'infini comme fini, sur la base 4 .

Alain Pontaut, critique littéraire, est aussi l'auteur de romans, d'une pièce de théâtre et de recueils de poèmes. Le Train des limbes ${ }^{3}$ est un mince ouvrage qui réunit une suite poétique en vers libres, celle qui donne son titre à l'ensemble; et des «Sonnets noirs» qui respectent à peu près les règles de la versification traditionnelle.

On ne peut nier le charme de certains poèmes, de certains vers plutôt car l'émerveillement se soutient rarement tout au long de la lecture. Pas de niaiserie, certes: mais le "message" (car il y en a un) n'a rien de bien neuf et consiste à affirmer, d'une part, la fatale décomposition de tout, à commencer par soi (mes membres éclatés / mon cadavre replet / mes entrailles grouillant de vers; ou encore: L'immense geignement de la misère humaine), et à surimposer un happy end, ce qui ne va pas toujours sans contorsion:

Je voulais au néant de nos chansons immondes

Crier que l'homme doit réinventer le monde

Et se dresser vaincu enfin mais accompli 
Vaincu mais accompli: je ne connais guère d'oxymore plus idéaliste, plus artificiellement conçu pour neutraliser les incompatibles. Trope bon pour les limbes.

D'autres vers, heureusement, proposent de fraîches images, pas fracassantes mais séduisantes. En voici, hors contexte, pour le choc suggestif des mots: Avec nos yeux de soie pour étancher l'azur, Aux creux secs du sel, Ensorcèlent (sic) de vent les destins innocents, Dans l'aube de papier nos extases de roi. Les influences de Baudelaire, Nerval, Rimbaud et même Mallarmé sont sensibles dans les sonnets, qui évoquent inévitablement d'autres temps, un autre rapport au réel.

Les limbes, ce sont ces bords du monde, entre vie et mort, où le poète situe sa revanche toute rhétorique sur les déboires de l'existence. Là, nos âmes vocalisent, ce qui est assez bien vu. Le vrai chant viendra peut-être plus tard, après les exercices de style.

Du style, on en souhaiterait à Janou Saint-Denis, qui s'en passe volontiers. Ce qui lui importe, c'est la passion: celle de vivre, et celle de promouvoir la poésie, quelle qu'elle soit. On connaît l'animatrice, irremplaçable. On peut maintenant connaître mieux le poète, qui a ses mérites. L'écriture n'est jamais, chez elle, dissociée des combats de l'existence, qu'il s'agisse des amours tumultueuses ou tragiques, des amitiés, des révoltes quotidiennes. À la Roue du feu secret ${ }^{4}$, Janou flambe et se distribue en cent et un textes de circonstance, porteurs de la même bruyante énergie. Son art poétique est affiché dès la première page:

poèse des mots à dire peau à l'aise
des maux dits maudit
être bien dans sa peau
se mouvoir se palper s'entendre
se jouir

Le mot poèse reprend le titre, «Peau aise", qui en est l'analyse anticipée et qui montre bien que l'écriture est affaire de corps, non de jolis vocables. Selon la logique facile de l'homophonie, les mots sont des maux; et la tradition à vénérer est celle des grands maudits - on pense à Rimbaud, Artaud, Gauvreau. Les damnés seuls ont la jouissance de leur vie car ils savent expulser d'eux-mêmes les maux, éprouver ce qui s'appelle plus loin L'ORGASME DEFFLAGRATEUR / (qui) ouvre et bascule / emmêle les cours / galvanise les sangs.

Malgré leur côté éloquent et brouillon, les textes de la reine des nuits de poésie de Montréal (Jean-Paul Daoust) ont de quoi faire rêver, comme toute expression populaire et féminine d'une authentique liberté. 
je crois

je sais que la littérature

est une perte absolue

écrit Michel Gay dans son élégant petit livre intitulé Mentalité, détail 5 . Et il s'arrange pour limiter les dégâts le plus possible, en recourant à l'écriture minimum. La première section contient treize pages-poèmes où quelques mots seulement, sur une, deux ou trois lignes, quatre exceptionnellement, sollicitent l'attention. On se dit, comme Pascal scrutant la nuit: Le silence éternel de ces espaces infinis m'effraie. On a froid devant le sens qui a ainsi fait retrait au fond du cosmos et ne subsiste que sous l'apparence d'une ironique poignée d'étoiles. La forme elle-même a disparu - car elle ne peut se soutenir que du matériau verbal, la pauvre. Ici, tout dit la rareté du matériau, la rareté. La forme n'a laissé pour toute trace que d'occasionnels filets sous lesquels se blottissent de frileuses formations verbales. Ce qui est atteint, en fait, c'est le texte: on est dans la performance orpheline, hors compétence. Le dire ne signifie rien d'autre que dire.

Quand, plus loin, Michel Gay revient à une certaine densité verbale, c'est pour parodier le texte, non le produire. Il écrit des poèmes qui auraient l'air de poèmes, avec du style et tout. Mais n'allez pas croire à quelque sincérité d'énonciation. La sincérité, elle reste en dehors du texte, dans le parti désespéré d'écrire le minimum, de saccager les chances d'un accomplissement de l'écriture. Je cite:

\section{argile d'écho aux paupières rouille \\ mots de trop d'étoiles \\ refilées à jamais salive \\ étale (tout) \\ mêlée au même basculé de mort \\ au creux maintenant de la mort}

Le poème crée une atmosphère métaphysique, avec ces mots de trop d'étoiles et cette mort deux fois en position finale. Une gravité - effet de langage - et une concision classique font l'attrait du discours. Mais rien du poète, rien du vivre n'est risqué dans les mots. Tout est perte déjà ; le poète seul s'en tire indemne, merveilleusement.

Roger des Roches, lui, se met à nu. Le Soleil tourne autour de la Terre ${ }^{6}$ n'est pas un poème cosmologique, comme on s'en doute, mais une fantaisie érotique en quarante-huit tableaux, suivis de "Preuves de la sphéricité de la Terre" qui met quelques points sur les $\mathrm{i}$.

Infantilisme, égocentrisme, machisme sont les supports du fantasme sans cesse renouvelé par lequel le poète règle son rapport à l'autre. "L'autre est un jouet ", tel est le titre de neuf des textes, qui forment une suite à l'intérieur de la suite. Cela veut dire que tout est jeu, que la femme (de préférence très 
jeune) est pur objet de conquête ou de possession, que le moi consent à devenir une poupée à mon tour s'il le faut, le jouet qui incite à ne pas poser aucune question. Vraiment le Soleil tourne autour de la Terre puisque toutes viennent à moi au simple appel, comme autant de répliques de la Mère bonne. Le poète dès lors prétend vivre immensément libre de tout souci, immensément insouciant, du seul désir enfantin de satisfaire et d'être satisfait.

Il y a beaucoup de fraîcheur dans cette revendication de valeurs contraires aux conceptions convenues de la maturité. Des Roches provoque en affichant une attitude superbement adolescente, du reste dénuée de tout mysticisme, simplement un émerveillement de collégien devant l'enchantement du corps. Son infini égoïsme trouve là son pardon, d'ailleurs il ne s'agit que de fantasmes, d'écriture. Le poème donne quelque perspective à cette petite science des trous $d u$ corps en évoquant le fantôme métaphysique d'un Événement difficile dont rien, au fil des pages, ne permet de préciser la nature. Voilà donc un peu de mystère pour saupoudrer le matérialisme décidé du récit, lequel court à travers les prétendus "poèmes» (en prose) et raconte une randonnée en automobile vers les plages de la Nouvelle-Angleterre, autour desquelles tourne effectivement le soleil des Québécois.

La femme n'ayant aucune autonomie, des Roches, un peu las de ses propres défauts, doit lui souffler son rôle: Je suis encombrant. / Accuse-moi. Accuse-moi!

À lire pour se reposer du féminisme. Mais aussi parce que des Roches est un écrivain plein d'inventions, de présence, d'humour. Il me semble seulement guetté de plus en plus par le prosaîsme, où se dévoie la grande force expressive qui faisait le prix de l'Imagination laïque ou de Poème, attention!

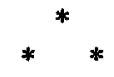

En 1980, lors du fameux colloque de la NBJ sur la Nouvelle Écriture, il était déjà question d'un retour au lisible et des dangers qu'il représentait. Il aura fallu quelques années pour que l'un et les autres se matérialisent vraiment. Les dangers, on les observe à l'œil nu dans un recueil tel que Bacilles de tendresse, de Pozier, dont j'ai parlé déjà ${ }^{7}$. D'autres poètes, au contraire, savent tirer parti de la consigne du jour, leur inspiration étant spontanément accordée à un type de discours qui appelle la consommation immédiate. Je pense à Jean-Paul Daoust et à son Dimanche après-midi ${ }^{8}$ (dont le titre en rappelle quelques-uns d'André Roy). En lisant ce recueil, on a le sentiment d'une parfaite harmonie des composantes formelles et sémantiques, sur le mode mineur comme on disait à l'époque où les bibliothèques avaient plusieurs rayons et les lyres, des demi-tons.

L'unité du recueil est assurée par la récurrence des mêmes thèmes: l'ennui, l'échec, le désarroi, la quotidienneté de tout le monde et, au fond de tout cela, l'écriture de la mort, plus précisément le souvenir du père disparu. Les mots dimanche(s) après midi reviennent dans presque tous les poèmes, 
dont la longueur varie entre cinq et quinze vers (quelques lignes de prose viennent parfois commenter une circonstance de lieu ou de temps).

Harmonie, disais-je, de la «forme» et du «sens». La poésie est là, mais c'est une poésie limitée, dénuée de fulgurances. Les images, peu nombreuses, ont une intelligibilité immédiate. La connotation n'est pas l'effet direct du langage mais surgit plutôt de l'intervalle entre les énoncés, en eux-mêmes parfaitement clairs:

La philosophie de l'heure

Le poète se demande si tout le monde pense

Nucléaire le désarroi

la même chose

Les mots organisent de nouveaux pays (...)

A la dérive dans son histoire personnelle et dans une histoire collective qui ne présente pas plus d'intérêt, le poète conjure comme il peut l'ennui, lequel recèle heureusement, en creusant un peu, quelques ressources d'angoisse et d'horreur.

Hors champ, d'Hélène Dorion ${ }^{9}$, présente de belles qualités d'écriture. Peu ou pas de facilités, même si le registre thématique les favorise. Le poète dit ses amours quotidiennes. La quotidienneté est ici l'objet d'une véritable interrogation, dans le contexte de la relation à l'être aimé. Les objets, qui sont souvent fatals à la poésie, sont peu présents; le corps, en revanche, l'est dans toutes ses modalités, malgré une tendance à l'abstraction.

Cette poésie, en effet, bien que simple, atteint la densité qui lui est propre par une certaine mise à distance du référent. Peut-être le titre trouvera-t-il là son explication; l'écriture met «hors champ» les formes du présent:

Mes doigts sur ce mur

quelques points quelques traits

dissimulent l'impartageable

poussière d'une vie

Les doigts sont réduits, par l'image, à des êtres géométriques (points, traits); l'adjectif abstrait (impartageable) est mis en évidence par le rejet du substantif concret (poussière) au début du vers suivant. Cela dit, il n'y a pas éviction du réel ou du corps mais plutôt déplacement, au profit de la syntaxe du vécu.

Les reproches que je serais tenté d'adresser au poète visent la relative monotonie de l'inspiration, qui ne change guère tout au long du recueil, et la complaisance dans la rhétorique (les belles formules). Il y a souvent un décalage entre l'idée et son expression quelque peu ampoulée: Dans l'étau de tes bras, n'ai-je pas oublié quelques muscles? Défauts de surface. Les livres à 
venir seront sans doute l'occasion d'un épanouissement car Hélène Dorion a manifestement quelque chose à dire. Elle a de plus le sens du travail poétique. Il ne lui manque qu'un brin d'assurance et la conquête de ses terres propices.

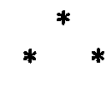

Ça crépite, la poésie de Rosie Harvey. Le Prix du vivant ${ }^{10}$ pourrait faire penser à Rimbaud - mais un Rimbaud femme, et capable de sentiments. Comme dans les Illuminations, l'isotopie du texte est fragile, l'image fuse dans toutes les directions. Par exemple: J'habite un trou aux accès bouchés. J'habite la forêt, la vierge, l'amazone, la jungle et je hurle la nuit qui est faite pour ça. Voilà qui roucoule et qui ronronne, la peau claque, la chair s'ébroue. On voit qu'il s'agit d'instituer, tout en le contrôlant, un arbitraire. L'enchaînement des idées repose sur une logique de l'imprévu; des propositions brèves, ramassées, se succèdent brutalement.

Cette intensité du discours masque mal l'absence d'un propos d'ensemble bien défini. Il me semble que, faute d'argument, le poème contient peu de développement. On saute d'une idée à l'autre sans qu'aucune prenne véritablement forme. Dans les lignes que j'ai citées, une première représentation de l'espace habité (trou aux accès bouchés) cède la place à une deuxième (forêt) et on est tout de suite catapulté vers la nuit, la peau, la chair. Tout sert à exprimer tout, et malgré sa minceur, le recueil nous semble long: le discours, trop ouvert à la totalité de ses possibles, fait du surplace. Des thèmes comme "l'hypothèse" - celle de la mort, celle de la vie - n'arrivent guère à articuler le discours et le tirer de son immobile frénésie.

Du talent plein les dards, est-il écrit quelque part. C'est dans le détail de l'expression que Rosie Harvey excelle, sans aucun doute. Ses images sont un essaim de frelons, de petites brûlures gratuites! Il faudrait cependant passer aux agressions majeures, faire du texte tout entier l'instrument d'un triomphe sur le lecteur - ou d'une défaite encore plus profitable.

France Daigle, auteur de trois livres aux éditions d'Acadie de Moncton, fait paraître une fort originale plaquette à la NBJ: Variations en B et $K^{\prime \prime}$. Le titre suggère peut-être une expérimentation formelle, abstraite et froide du langage. En fait, il n'en est rien. L'humour et la simplicité flanquent l'intelligence et la rendent parfaitement attrayante!

Le discours des Variations ne relève pas de la poésie à proprement parler mais de cette pratique textuelle dont on parlait à Tel Quel et qui s'imposera peut-être un jour comme un genre autonome, distinct de la poésie comme de la prose (narrative, didactique...).

Les effets de sens sont ici obtenus de la réunion de dispositifs textuels hétérogènes. En haut, en italique, une sorte de relation de voyage qui nous transporte indécidablement dans l'ici (un parc de camping qui pourrait se 
situer autour de Bouctouche ou de Kouchibougouac) ou l'ailleurs (dans le désert, au milieu des Kurdes ou des Bédouins). Le récit, tout à fait fragmentaire, confine plutôt à la note historique ou autobiographique. En bas, une autre note composée en tout petits caractères offre le commentaire d'une ou de quelques photos fictives, censées illustrer le livre. Les rapports entre les deux formations textuelles sont souvent lointains, voire inexistants; de là résulte un efficace climat de parodie. La gratuité du jeu est cependant réduite par la troublante abondance des noms propres, et même communs, dont l'initiale est soit B (Bouctouche, Bédouins...), soit K (Kurdes, Kouchibougouac...), et qui se rapportent sans arbitraire apparent aux deux isotopies référentielles que sont l'ici et l'ailleurs.

Le but de l'opération, qui est beaucoup plus qu'un exercice de style, est peut-être de montrer ce qu'il y a de familier, de bon (B) au cœur de l'ailleurs (K) et inversement, d'ouvrir au monde la maison (la lettre B dans l'alphabet phénicien représentait la maison, c'est-à-dire, pour les modernes, la personnalité).

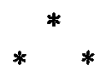

Les poèmes que réunit André Roy dans Action Writing ${ }^{12}$ appartiennent à plusieurs époques, assez régulièrement distribuées entre 1973 et 1984 . Les premiers relèvent d'une esthétique "formaliste", les derniers font retour au "lisible» (les deux notions sont aussi problématiques l'une que l'autre). $\mathrm{Ce}$ qui frappe cependant, à travers les différences d'écriture, c'est la permanence d'un style que je qualifierais de classique. Il y a une absence remarquable de pathétique chez André Roy, alors même qu'il célèbre la passion. La passion est avant tout celle du corps, et non des sentiments comme l'affirme le texte en page couverture (la passion des sentiments étant, du reste, une formule passablement redondante). La sexualité est le filtre tout-puissant de l'affectivité et empêche les égarements, les désespoirs, les chavirements de l'amour impossible. Elle impose à l'existence sa mesure, on pourrait dire sa raison.

Et, comme le classicisme est action, la poésie d'André Roy se nourrit essentiellement de performances et d'événements. La référence à Jackson Pollock, l'initiateur de l'action painting, dont sept tableaux sont l'objet de paraphrases (au sens où Malherbe paraphrasait les Psaumes), montre assez que l'écriture valorise l'acte même par lequel elle prend possession d'un champ qu'elle sature de signifiance:

\section{(...) La couleur revient}

d'au delà d'une forêt inaudible: tout ce qui

descend du ciel est flèche, force, histoire

de la peinture. Tout ce qui rêve en nous

est visible au dehors, dedans et dehors.

Ne rien laisser aux ténèbres intimes, tout manifester de ce que produit l'inconscient (forêt inaudible), le traduire immédiatement en histoire de la peinture, comme Phèdre analyse sa passion et produit l'aveu qui la rature 
(tout ce qui descend du ciel est flèche, force), voilà l'essence même du classicisme. On ne sait trop ce qu'une telle poétique, employée à la célébration de la femme, pourrait produire aujourd'hui; André Roy évoque les beaux objets de son désir avec une retenue qui n'exclut ni l'ivresse, ni la crudité, et qui renouvelle en profondeur le discours amoureux.

Toute poésie est agression. Ou bien elle heurte, par une transparence décevante, notre goût profond de l'inédit, fondé sur le besoin de refaire notre rapport au monde et au langage. Ou bien sa gratuité déconcerte, met à mal la raison. Entre ces deux tendances contraires, les bons poètes savent inventer, toujours à nouveaux frais, l'équilibre nécessaire.

Hélas, ce n'est pas le cas de Jocelyne Felx dans Nickel-Odéon ${ }^{13}$. L'auteur d'Orpailleuse ${ }^{14}$ avait pourtant séduit par un langage plein de trouvailles. Mais ici, l'arbitraire du discours l'emporte de loin sur les chances de sa réception. Est-ce excès de richesse? Toutes sortes de voix se mêlent: philosophie (l'alternance du pour-soi et de l'en-soi germe des rêves...), politique (Il ressemble à Mobutu content de son coffre dans une banque suisse), conte populaire (que ne sont-ils-t-elles filles et fils d'ogres et d'ogresses pour dévorer les loups), sociologie, psychologie, communications, sciences naturelles, etc. Grande abondance de mots rares, bondance! On dirait un journal du matin pour lexicographes. La vie est un ersatz pour les mots. Cette phrase, qui s'applique à quelqu'un d'autre, vaut aussi pour l'auteur.

Sans compter le gynécocentrisme.

Les sujets, pourtant, ont quelque chose de très simple - de trop simple peut-être? «Salines» propose de s'abandonner au mouvement du fleuve, de la mer. Cela devient: Laver les crises d'infaillibilité de cet unique et exclusif amour. Noir acier morganatique. Après quoi tout se complique à l'infini.

Heureusement, dans la veine tendre, le "Poème à Delphine» contient de ravissants passages. Ces réussites sont sans doute le fruit de l'intervention décidée de la prose dans les affaires du poème car la poésie comme telle, on l'a vu, ne peut être que rébarbative. Agression du second type: celui qui tarabuste l'intellect. La prose y introduit le référent, c'est-à-dire une certaine stabilité du sens, où la suggestion peut faire son nid.

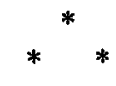

Il me reste à signaler, dans la collection "Typo", la réédition de l'intégrale des poèmes de Jean-Guy Pilon, Comme eau retenue ${ }^{15}$, et celle des trois admirables recueils de Paul Chamberland, Terre Québec, l'Afficheur hurle.et I'Inavouable (avec une préface de votre serviteur, comme on dit) ${ }^{16}$. Je signale aussi, avec bien du retard, le numéro spécial de Dalhousie French Studies qu'Eva Kushner et Michael Bishop ont consacré à la Poésie québécoise 
depuis $1975^{17}$; la publication réunit des essais, des témoignages et un riche éventail d'inédits. Enfin, j'attire l'attention sur une nouvelle revue de poésie, Modern'18, plus mince encore que Lèvres urbaines ${ }^{19}$ (le premier numéro compte 12 pages). Son directeur, Lucien Francoeur, ne semble pas tout à fait convaincu de sa nécessité mais termine son "éditorial" en se souhaitant Bonne route!. A-t-il seulement besoin de lecteurs?

1. Normand de Bellefeuille, Lascaux, Montréal, les Herbes rouges, 1985, 160 p.

2. Normand de Bellefeuille, le Livre du devoir, Montréal, les Herbes rouges, 1983, 100 p.

3. Alain Pontaut, le Train des limbes, Montréal, Leméac, 1985, 60 p.

4. Janou Saint-Denis, la Roue du feu secret, Montréal, Leméac, 1985, 302 p.

5. Michel Gay, Mentalité, détail, Montréal, NBJ, 1986, n.p.

6. Roger des Roches, Le Soleil tourne autour de la Terre, Montréal, les Herbes rouges, $1965,74 \mathrm{p}$.

7. Cf. «Le lisible et ses ombres", chronique de poésie, Voix et images, vol. XI, no 3, printemps 1986.

8. Jean-Paul Daoust, Dimanche après-midi, Trois-Rivières, Écrits des Forges, 1985, $54 \mathrm{p}$.

9. Hélène Dorion, Hors champ, Saint-Lambert, le Noroît, 1985, $110 \mathrm{p}$.

10. Rosie Harvey, le Prix du vivant, Trois-Rivières, Écrits des Forges, 1985, 50 p.

11. France Daigle, Variations en B et K, Montréal, NBJ, 1985, 44 p.

12. André Roy, Action Writing, Montréal, les Herbes rouges, 1985, 112 p. Prix du gouverneur général en poésie, 1985.

13. Jocelyne Felx, Nickel-Odéon, Saint-Lambert, le Noroit, 1985, 174 p.

14. Jocelyne Felx, Orpailleuse, Saint-Lambert, le Noroit, 1982, 70 p. Cf. mon compte rendu dans "Des femmes et des mots», Voix et images, vol. VIII, no 3, printemps 1983. p. 504.

15. Jean-Guy Pilon, Comme eau retenue, Montréal, l'Hexagone, "Typo», 1985, 234 p.

16. Paul Chamberland, Terre Québec suivi de l'Afficheur hurle et de l'Inavouable, Montréal, l'Hexagone, «Typo», 1985, $288 \mathrm{p}$.

17. La Poésie québécoise depuis 1975 , sous la direction d’Éva Kushner et de Michael Bishop, Dalhousie French Studies, Dalhousie, s.d., 202 p.

18. Modern', vol. I, no 1, Montréal, 1985, $12 \mathrm{p}$. Le numéro contient des poèmes de Claudine Bertrand, Jean-Marc Desgent, Françoise Favretto, Lucien Francœur, Michel Gay, Philippe Marchal, Bernard Pozier, Maurice Roche, Philippe Soupault et Marc Villard.

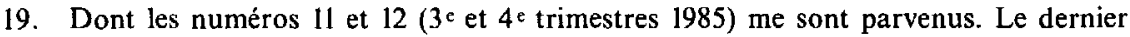
constitue une plaquette en collaboration, signée Herménégilde Chiasson et Gérald Leblanc. 


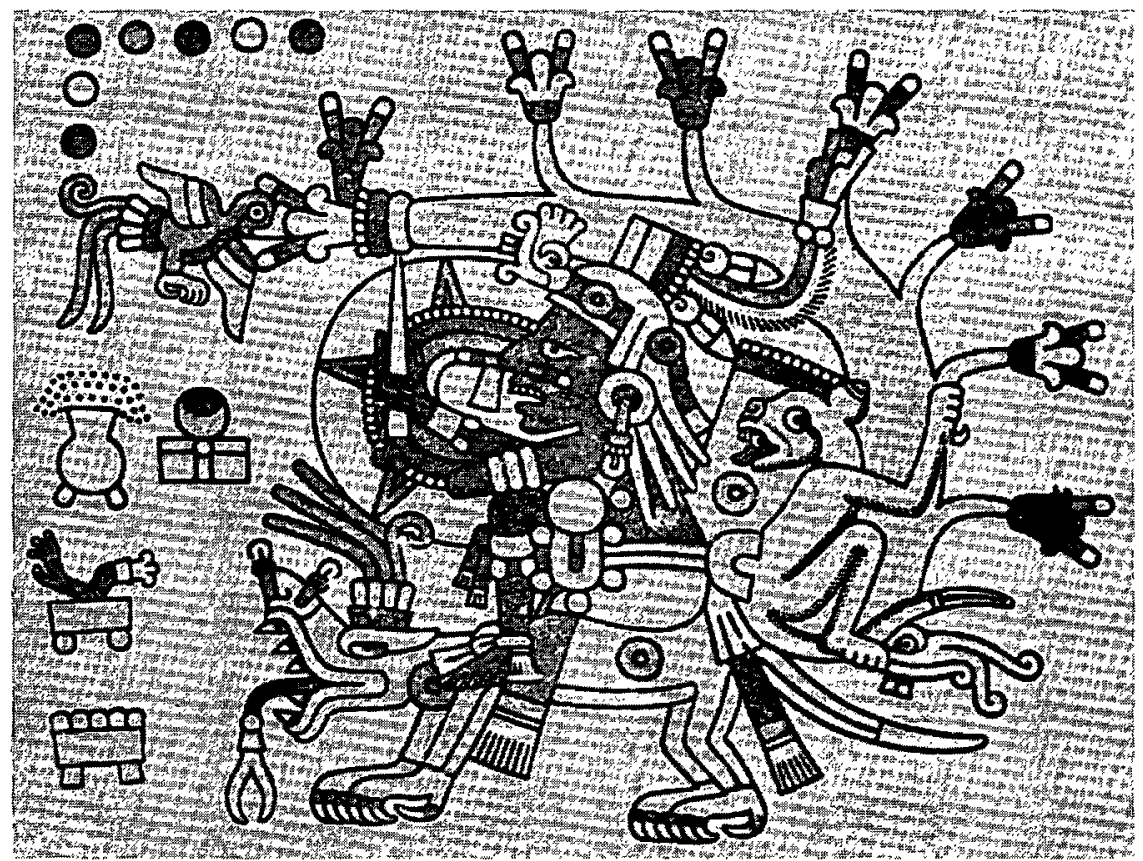

